\title{
Three different effects of the same quantum nature
}

\author{
Michael A. Ivanova,* \\ a Physics Dept., Belarus State University of Informatics and Radioelectronics, \\ 6 P. Brovka Street, Minsk, Belarus \\ E-mail: ivanovma@tut.by
}

In the model of low-energy quantum gravity by the author the cosmological redshift and additional dimming of remote objects may be interpreted as results of scattering of photons on gravitons of the background. A tentative detection of a diffuse cosmic optical background by the New Horizons mission may be connected with non-forehead collisions of photons with gravitons, too. The conjecture about the local quantum-gravitational nature of the redshift may be verified in a ground-based laser experiment partly using advanced LIGO technologies.

The European Physical Society Conference on High Energy Physics (EPS_HEP2021) 26-30 July 2021

Online conference, jointly organized by Universität Hamburg and the research center DESY 


\section{Introduction}

The main conjecture of the model of low-energy quantum gravity [1,2] is the existence of the background of super-strong interacting gravitons. Its temperature $\mathrm{T}$ defines values of the Newton and Hubble constants, and the ones may be computed. The inverse-square law of classical gravity describes the main quantum effect of this model. The Hubble constant is not connected here with any expansion of the universe, but only with energy losses of photons due to forehead collisions with gravitons of the background that causes redshifts of spectra of remote galaxies. The additional effect of decreasing a number of photons in a propagating beam due to non-forehead collisions with gravitons can explain the discovered in 1998 additional dimming of remote sources [3, 4]. These scattered photons will be registered by a remote observer as going from nowhere; it may be interpreted as a diffuse cosmic background. A tentative detection of a diffuse cosmic optical background [5] may be connected with this light-from-nowhere effect of the model.

\section{The light scattering on gravitons in low-energy quantum gravity}

Energy losses of photons due to forehead collisions with gravitons of the background lead to the geometrical distance/redshift relation of this model:

$$
r(z)=\ln (1+z) \cdot \frac{c}{H_{0}}
$$

where $\mathrm{H}_{0}$ is the Hubble constant, $\mathrm{c}$ is the velocity of light. We may introduce the Hubble parameter $\mathrm{H}(\mathrm{z})$ in the following manner:

$$
d z=H(z) \cdot \frac{d r}{c}
$$

to imitate the local Hubble law. Taking a derivative $\frac{\mathrm{dr}}{\mathrm{dz}}$, we get in this model without expansion for $\mathrm{H}(\mathrm{z})$ :

$$
H(z)=H_{0} \cdot(1+z) .
$$

The Hubble parameter $\mathrm{H}(\mathrm{z})$ of this model is a linear function of $\mathrm{z}$ (as well as in the $\mathrm{R}_{\mathrm{h}}=\mathrm{ct}$ cosmological model [6]), that is in a big discrepancy with $\Lambda$ CDM. As it was shown, this function fits available observations of $\mathrm{H}(\mathrm{z})$ very well $[6,7]$.

There exists an additional effect of decreasing a number of photons in a propagating beam due to non-forehead collisions with gravitons which allows to get along without any dark energy in interpreting cosmological observations. These two effects give the luminosity distance/redshift relation of the model:

$$
D_{L}(z)=c / H_{0} \cdot \ln (1+z) \cdot(1+z)^{(1+b) / 2},
$$

where the "constant" $\mathrm{b}$ belongs to the range $0-2.137$ ( $b=\frac{3}{2}+\frac{2}{\pi} \simeq 2.137$ for very soft radiation, and $\mathrm{b} \rightarrow 0$ for very hard one). This relation fits cosmological observations of remote sources very well without dark energy [2].

Non-forehead collisions of photons with gravitons cause the deviation of some photons from an initial direction of propagation. These photons will be registered by a 
remote observer as going from nowhere even by small, but finite, angles of deviation. After multiple non-forehead collisions, the direction of propagation of these photons may be essentially different from the initial one. The scattered photons will be redshifted proportionally to passed random distances. Because of this randomness of angles of scattering and passed distances, it is difficult to compute the sky brightness in the optical range, for example, due to this light-from-nowhere effect. It is necessary to know the ratio $\delta(\mathrm{z})$ of the scattered flux to the the remainder reaching the observer, and, at least, the light flux of galaxies and their number counts by different redshifts. To evaluate how big is the ratio $\delta(\mathrm{z})$, we can compute the flux, where $\mathrm{L}$ is the luminosity, $\mathrm{D}_{\mathrm{L}}(\mathrm{b}, \mathrm{z})$ and $\mathrm{D}_{\mathrm{L}}(0, \mathrm{z})$ are luminosity distances by $\mathrm{b} \neq 0$ and $\mathrm{b}=0 . \Phi_{0}(\mathrm{z})$ corresponds to the absence of nonforehead collisions. Then the ratio may be defined as:

Using Eq. 4 we get:

$$
\delta(z) \equiv \frac{\Phi_{0}(z)-\Phi(z)}{\Phi(z)} .
$$

$$
\delta(z)=(1+z)^{b}-1 .
$$

We have by $\mathrm{b}=2.137: \delta(0.4)=1.05, \delta(1)=3.34, \delta(2)=9.46$. But a problem to compute the sky brightness remains open: one should take into account the randomness of angles of scattering and passed distances of photons in some probabilistic manner.

\section{How to verify the quantum redshift mechanism}

The main conjecture of this approach about the quantum nature of redshifts may be verified in a ground-based laser experiment. To do it, one should compare spectra of laser radiation before and after passing some distance $\mathrm{l}$ in a high-vacuum tube [2]. The temperature $\mathrm{T}$ of the graviton background coincides in the model with the one of CMB. Assuming $\mathrm{T}=2.7 \mathrm{~K}$, we have for the average graviton energy: $\bar{\epsilon}=8.98 \cdot 10^{-4} \mathrm{eV}$. Because of the quantum nature of redshift, the satellite of main laser line of frequency $v$ would appear after passing the tube with a redshift of $10^{-3} \mathrm{eV} / \mathrm{h}$, and its position should be fixed. It will be caused by the fact that on a very small way in the tube only a small part of photons may collide with gravitons of the background. The rest of them will have unchanged energies. The center-of-mass of laser radiation spectrum should be shifted proportionally to a photon path. Due to the quantum nature of shifting process, the ratio of satellite's intensity to main line's intensity should have the order: $\sim \frac{\mathrm{h} v}{\bar{\epsilon}} \frac{\mathrm{H}_{0}}{\mathrm{c}}$ l. Given a very low signal photon number frequency, one could use a single photon counter to measure the intensity of the satellite line after a narrow-band filter with filter's transmittance $\mathrm{k}$. If $\mathrm{q}$ is a quantum output of a photomultiplier cathode, $\mathrm{f}_{\mathrm{n}}$ is a frequency of its noise pulses, and $\mathrm{n}$ is a desired signal-to-noise ratio, then an evaluated time duration $\mathrm{t}$ of data acquisition would be equal to:

$$
t=\frac{(\bar{\epsilon} c n)^{2} f_{n}}{\left(H_{0} q k P l\right)^{2}}
$$

where $\mathrm{P}$ is a laser power. Assuming for example: $\mathrm{n}=10, \mathrm{f}_{\mathrm{n}}=10^{3} \mathrm{~s}^{-1}, \mathrm{q}=0.3, \mathrm{k}=0.1$, $\mathrm{P}=200 \mathrm{~W}, \mathrm{l}=300 \mathrm{~km}$, we have the estimate: $\mathrm{t} \approx 3 \cdot 10^{3} \mathrm{~s}$. Such the value of $\mathrm{l}$ may be 
achieved if one forces a laser beam to whipsaw many times between mirrors in the vacuum tube with the length of a few kilometers.

The advanced LIGO detectors [8] have many technological achievements needed to do the described experiment: stable powerful lasers and input optics, high-vacuum tubes with optical resonator that multiplies the physical length by the number of round-trips of the light, mirror suspension systems with actuators. Some parameters of LIGO systems are of the same order as in the considered example. If one constructs a future LIGO detector with some additional equipment, the verification of the redshift mechanism may be performed in parallel with the main task or during a calibration stage of the detector.

\section{Conclusion}

The described three effects of low-energy quantum gravity give us a possibility to interpret well known redshifts of remote objects and their additional dimming discovered in 1998, together with a tentative existence of a diffuse cosmic optical background [5], in a very simple unified manner. This description makes unnecessary any expansion of the universe and dark energy. The model of low-energy quantum gravity is able to fit available observations of the luminosity distance $D_{L}(z)$ and of the Hubble parameter $H(z)$ very well. One should carry out the precise ground-based laser experiment to verify the basic conjecture of this model.

\section{References}

[1] M.A. Ivanov, Gravitons as super-strong interacting particles, and low-energy quantum gravity. In the book "Focus on Quantum Gravity Research", Ed. D.C. Moore, Nova Science, NY - 2006 - pp. 89-120; [hep-th/0506189].

[2] M.A. Ivanov, Selected papers on low-energy quantum gravity. [http://ivanovma.narod.ru/selected-papers-Ivanov2018.pdf].

[3] A.G. Riess, et al., Observational Evidence from Supernovae for an Accelerating Universe and a Cosmological Constant. AJ 1998, 116, 1009.

[4] S. Perlmutter et al., Measurements of $\Omega$ and $\Lambda$ from 42 High-Redshift Supernovae. ApJ 1999, 517, 565.

[5] T.R. Lauer et al., (2011). New Horizons Observations of the Cosmic Optical Background. [arXiv:2011.03052v2 [astro-ph.GA]].

[6] F. Melia and M.K. Yennapureddy, Model selection using cosmic chronometers with Gaussian Processes. JCAP 02(2018)034; [arXiv:1802.02255v2 [astro-ph.CO]].

[7] M.A. Ivanov, Low-energy quantum gravity and cosmology without dark energy. Advances in Astrophysics 2019, 4, No. 1, pp. 1-6.

[8] B.P. Abbott et al., GW150914: The Advanced LIGO Detectors in the Era of First Discoveries. Phys. Rev. Lett. 2016, 116, 131103; [arXiv:1602.03838 [gr-qc]]. 